\title{
Commentary: Does Severity of Alzheimer's Disease Contribute to Its Responsiveness to Modifying Gut Microbiota? A Double Blind Clinical Trial
}

\author{
Friedrich Leblhuber ${ }^{1}$, Kostja Steiner ${ }^{1}$, Burkhard Schuetz ${ }^{2}$ and Dietmar Fuchs ${ }^{3 *}$ \\ ${ }^{1}$ Department of Gerontology, Neuromed Campus, Kepler University Clinic, Linz, Austria, ${ }^{2}$ Biovis Diagnostik MVZ GmbH, \\ Limburg, Germany, ${ }^{3}$ Division of Biological Chemistry, Biocenter, Innsbruck Medical University, Innsbruck, Austria, ${ }^{4}$ Division of \\ Medical Biochemistry, Biocenter, Innsbruck Medical University, Innsbruck, Austria
}

Keywords: Alzheimer's dementia (AD), probiotic supplementation, inflammation, TYM test, biomarkers

\section{A Commentary on}

Does Severity of Alzheimer's Disease Contribute to Its Responsiveness to Modifying Gut Microbiota? A Double Blind Clinical Trial

by Agahi, A., Hamidi, G. A., Daneshvar, R., Hamdieh, M., Soheili, M., Alinaghipour, A., et al. Front.

OPEN ACCESS

Edited by:

Tibor Hortobágyi,

University of Debrecen, Hungary

Reviewed by:

Kristyn Elizabeth Sylvia Johns Hopkins University,

United States

*Correspondence:

Dietmar Fuchs

dietmar.Fuchs@i-med.ac.at

Specialty section:

This article was submitted to

Neurodegeneration,

a section of the journal

Frontiers in Neurology

Received: 09 January 2019 Accepted: 06 June 2019

Published: 05 July 2019

Citation:

Leblhuber F, Steiner K, Schuetz B and Fuchs D (2019) Commentary: Does

Severity of Alzheimer's Disease Contribute to Its Responsiveness to Modifying Gut Microbiota? A Double

Blind Clinical Trial.

Front. Neurol. 10:667.

doi: 10.3389/fneur.2019.00667
Neurol. (2018). 9:662. doi: 10.3389/fneur.2018.00662

A. Agahi and colleagues in their study evaluated the responsiveness of inflammatory and oxidative biomarkers to a 12 weeks probiotic treatment in Alzheimer disease (AD) patients (1). After assessment of cognitive and serum biomarkers pre- and post-treatment in 96 patients with severe $\mathrm{AD}$, they concluded that the cognitive Test Your Memory (TYM) test and markers like serum total antioxidant capacity, total glutathione, cytokines IL-6, IL-10, and TNF- $\alpha$, and 8-hydroxy-2' deoxyguanosine and plasma nitrite/nitrate were insensitive to probiotic supplementation. They concluded that severity of disease and time of administration seems to deeply affect results of treatment.

To evaluate the cognitive decline in their AD patients they applied the TYM test, which is described as suitable for detecting dementia earlier than the mini mental state examination (MMSE) (2). The TYM test is superior in discriminating between healthy controls and patients with mild cognitive impairment (MCI). To use the TYM test instead of the MMSE probably was the reason for the fact that they did not find any difference in cognition between the verum treated group and the placebo group of severely demented AD patients after 3 months of supplementation with probiotics. In Figure 2 as well as in Figure 3 of their study (1) they demonstrated a clearly significant difference between the TYM scores between controls and AD patients as well as between moderate $\mathrm{AD}$ and severe $\mathrm{AD}$ patients (both $p<0.0001$ ). Regarding the possible influence of probiotic supplements, they probably had achieved more conclusive results (a) using the MMSE test instead of the TYM test and (b) comparing also normal individuals vs. patients with mild or moderate AD. Dysbiosis of intestinal microbiota in the elderly may cause leaky gut which results in silent systemic inflammation. Subsequent neuroinflammation may be a fundamental part of the pathomechanism in the early and preclinical course of $\mathrm{AD}(3)$.

In our own study (4) in a group of $20 \mathrm{AD}$ patients with a more advanced stage of dementia (MMSE $18.5 \pm 7.7$ ), we did find changes of serum and fecal inflammatory parameters already after 4 weeks of supplementation of a multispecies probiotic (Omnibiotic Stress Repair ${ }^{\circledR}$, Allergosan Graz Austria). The preparation consists of Lactobacillus casei W56, Lactococcus lactis W19, Lactobacillus 
acidophilus W22, Bifidobacterium lactis W52, Lactobacillus paracasei W20, Lactobacillus plantarum W62, Bifidobacterium lactis W51, Bifidobacterium bifidum W23, and Lactobacillus salivarius W24 $\left(7.5 \times 10^{9}\right.$ intestinal bacteria/per day). Inflammation-associated fecal zonulin was found significantly declining $(p=0.01)$ and anti-inflammatory Faecalibacterium prausnitzii significantly increased $(p<0.001)$ after 4 weeks of probiotic supplementation. By contrast, serum inflammation marker neopterin as well as kynurenine increased $(p<0.05)$, probably pointing to an activation of macrophages/dendritic cells by the probiotic intervention. The increase of nitrite levels from $238 \pm 421$ (mean \pm SEM) to $1050 \pm 442 \mu \mathrm{mol} / \mathrm{L}$ was only slightly outside the level of significance $(\mathrm{U}=-1.423, p=0.078$; Mann-Whitney $U$-test). Notably, nitrite concentrations better than nitrite+nitrate levels mimic nitric oxide production (5). As a result, our recent study (4) shows-contrary to the series of Agahi et al.- that the supplementation of demented AD patients with a multispecies probiotic clearly influences gut bacteria composition. The correlation between neopterin concentrations and tryptophan breakdown as is reflected by the kynurenine to tryptophan ratio (Kyn/Trp) most probably was caused by the activation of macrophages due to probiotic supplementation for 4 weeks (4). We did not control the MMSE after such a short period of 4 weeks to avoid a measurement error due to the test-retest variability (6).

\section{REFERENCES}

1. Agahi A, Hamidi GA, Daneshvar R, Hamdieh M, Soheili M, Alinaghipour A, et al. Does severity of Alzheimer's disease contribute to its responsiveness to modifying gut microbiota? A double blind clinical trial. Front Neurol. (2018) 9:662. doi: 10.3389/fneur.2018.00662

2. van de Zande E, van de Nes JC, Jansen I, van den Berg MN, Zwart AF, Bimmel D, et al. The test your memory (TYM) test outperforms the MMSE in the detection of MCI and dementia. Curr Alzheimer Res. (2017) 14:598-607. doi: 10.2174/1567205013666161201202520

3. Caracciolo B, Xu W, Collins S, Fratiglioni L. Cognitive decline, dietary factors and gut-brain interactions. Mech Aging Dev. (2014) 136-7:59-69. doi: 10.1016/j.mad.2013.11.011

4. Leblhuber F, Steiner K, Schuetz B, Fuchs D, Gostner JM. Probiotic supplementation in patients with Alzheimer's dementia - An exploratory intervention study. Curr Alzheimer Res. (2018) 15:1106-13. doi: 10.2174/1389200219666180813144834

5. Kleinbongard P, Dejam A, Lauer T, Rassaf T, Schindler A, Picker O, et al. Plasma nitrite reflects constitutive nitric oxide synthase activity in mammals. Free Radic Biol Med. (2003) 35:790-6. doi: 10.1016/S0891-5849(03)00406-4

6. Doraiswamy M, Kaiser L. Variability of the mini mental state examination in dementia. Neurology. (2000) 54:1538-9. doi: 10.1212/WNL.54.7.1538-a
The question arises, why a 12 week's probiotic supplementation did not show effects neither on proinflammatory nor on anti-inflammatory markers in the patients studied by Agahi et al. (1). Was it the fact that they used only $3 \times 10^{9}$ bacterial strains containing 3 bacteria compared to $7.5 \times 10^{9}$ bacteria of 9 different strains supplemented daily in our study (4)?

The results of our exploratory study (4) can only be regarded as preliminary due to the small sample size and due to the lack of a placebo treated group. Larger populations including patients with MCI as well as cognitively normal subjects are necessary to show whether probiotics are able to influence cognitive function at the very beginning of pathogenic changes of neurodegeneration and $\mathrm{AD}$ dementia. Future intervention studies with prebiotics and probiotics should include measurement of lipopolysaccharide activity (7) and short chain fatty acids $(8,9)$ to elucidate their possible role in the pathogenesis of $\mathrm{AD}$ for better understanding the disease mechanisms. This would provide the evidence needed to create more effective therapeutic interventions in $\mathrm{AD}$.

\section{AUTHOR CONTRIBUTIONS}

All authors listed have made a substantial, direct and intellectual contribution to the work, and approved it for publication.

7. Jiang C, Li G, Huang P, Liu Z, Zhao B. The gut microbiota and Alzheimer's disease. J Alzheimers Dis. (2017) 58:1-15. doi: 10.3233/JAD-16 1141

8. Erny D, Hrabe de Angelis AL, Jaitin D, Wieghofer P, Staszewski O, David E, et al. Host microbiota constantly control maturation and function of microglia in the CNS. Nat Neurosci. (2015) 18:965-77. doi: 10.1038/n n. 4030

9. Sampson TR, Mazmanian SK. Control of brain development, function, and behavior by the microbiome. Cell Host Microbe. (2015) 17:565-76. doi: 10.1016/j.chom.2015.04.011

Conflict of Interest Statement: The authors declare that the research was conducted in the absence of any commercial or financial relationships that could be construed as a potential conflict of interest.

Copyright (C) 2019 Leblhuber, Steiner, Schuetz and Fuchs. This is an open-access article distributed under the terms of the Creative Commons Attribution License (CC $B Y)$. The use, distribution or reproduction in other forums is permitted, provided the original author(s) and the copyright owner(s) are credited and that the original publication in this journal is cited, in accordance with accepted academic practice. No use, distribution or reproduction is permitted which does not comply with these terms. 